\title{
Predict the Future Hospitalized Patients Number Based on Patient's Temporal and Spatial Fluctuations Using a Hybrid ARIMA and Wavelet Transform Model
}

\author{
Shundong Lei \\ Information Management Department, West China Second University Hospital, Sichuan University, Chengdu, China \\ Email: leishundong@ruoshui.me
}

How to cite this paper: Lei, S.D. (2017) Predict the Future Hospitalized Patients Number Based on Patient's Temporal and Spatial Fluctuations Using a Hybrid ARIMA and Wavelet Transform Model. Journal of Geographic Information System, 9, 456-465.

https://doi.org/10.4236/jgis.2017.94028

Received: May 28, 2017

Accepted: August 7, 2017

Published: August 11, 2017

Copyright $\odot 2017$ by author and Scientific Research Publishing Inc.

This work is licensed under the Creative Commons Attribution-NonCommercial International License (CC BY-NC 4.0). http://creativecommons.org/licenses/by-nc/4.0/

\begin{abstract}
Relative to hospitalized patient information, outpatient admission information is relatively simple. It only includes the patient admission time, place of residence and other information. Traditionally, the excavation of this information is not sufficient. However, when a large number of patients admitted time and residence information combined to consider, and add some data mining technology, some of the previously ignored regular information is likely to be found. Using 5 years of data mining research and admission data from a paediatric department at a large women's and children's hospital in China, we found important fluctuation rules regarding admissions using wavelet analysis on hospital admission data among different scales of cyclical fluctuations. Method: Seasonal distribution of patient number was analysed based on Haar wavelet transformation, and level 3 and level 2 of wavelets were extracted out to fit the data. The distribution function of hospitalized patients was visualized by kernel density estimation. Using linear regression and ARIMA (autoregressive integrated moving average model) predict the seasonally number of patients in the future. Results: The data analysis demonstrates the total surge of inpatients was decomposed into one mother wavelet and five small wavelets, each of which represents different time frequency. Besides, as distance from hospital increases, the number of patients decreased exponentially. The seasonal factors are the largest time factor influencing the number changes of patients. Conclusion: By wavelet analysis and the improved prediction model, we could make forecast on the future inpatient number trend and prove factors such as geographic position is influential on inpatient amount. Additionally, the concept of data mining based on spatial distribution and spectral analysis could be applied to other aspects of social management.
\end{abstract}




\section{Keywords}

Medical Resources, Data Mining, Multi-Scale, ARIMA, Wavelet Transform Spatial Distribution

\section{Introduction}

Pretending changes in patient number and observing spatio-temporal patient distribution have been fundamental problems for hospital management. Many studies indicate that the patient spatio-temporal distribution contains important management information. Until now, most researches about patient admission are focused on simulation of disasters and public security incidents [1]. Few studies have focused on distribution of emergency resources. Hung's group reported traffic accident black spots in Hanoi and Vietnam using linear regression to determine a relationship between the black spot and an emergency distribution [2] [3]. These studies help us understand medical resource distribution and demand, but often, clinical activity is more related to diagnosis and patient treatment, so patient allocation and fluctuation represent challenges to allocation of medical resources.

To create a systematic analysis of routine medical procedures, two dimensions must be considered: time and space. For time, patients admitted to hospitals are influenced by days, weeks, or seasons, epidemics, and economic development. The probability of a patient admission during a specific time period can prompt the next period of probability of the patient's admission, so sequence analysis tools are required to analyze patient's admittance and machine-learning can assist with this process.

For spatial dimensions, patients in different regions are likely to have different medical needs. Selvin's studies suggest that patients from proximal regions are likely to have common diseases and patients from remote regions are likely to have chronic or rare diseases [4]. Patients from remote regions often expect a diagnosis which is a challenge to treatment. If we could understand patient distribution in time and space, we could better allocate medical resources and create an intelligent hospital management system based on big data.

To build a contemporary distribution for the forecast, the relationship between influencing factors and spatio-temporal distribution of hospitalized patients must be understood. Factors that influence hospitalized patients vary from economic development, education, population structure, and distance to the hospital [5] [6]. Therefore, technology to assess hospital needs should integrate these for more accurate medical resource distribution.

Recently, machine leaning has been used to devise complex models and algorithms that permit predictive analytics that reveal data trend relationships. Data from pediatric outpatient services are simple, but contain complex information. As a result of chorismitization of many factors, each entry of a pediatric record contains potential factor information, so we can build a cascade of layers of non- 
linear processing units for feature extraction and transformation. Each successive layer uses the output from the previous layer as input. Algorithms may be supervised or unsupervised and applications include pattern analysis (unsupervised) and classification (supervised).

Wavelet analyzation is based on unsupervised learning of multiple levels of features or representations of data. Higher-level features derived from lower level features were used to form a hierarchical representation. These definitions have common multiple layers of nonlinear processing units and supervised or unsupervised learning of feature representations in each layer, with the layers forming a hierarchy from low- to high-level features [2]. The composition of a layer of nonlinear processing units used in a deep learning algorithm depends on the problem to be solved. Layers used in deep learning include hidden layers of an artificial neural network and sets of complicated propositional formulas [3]. They may also include latent variables organized layer-wise in deep generative models such as the nodes in Deep Belief Networks and Deep Boltzmann Machines. Overall, with these methods, multivariate analysis of the whole system can forecast future development and provide solutions for admission data (Figure 1).

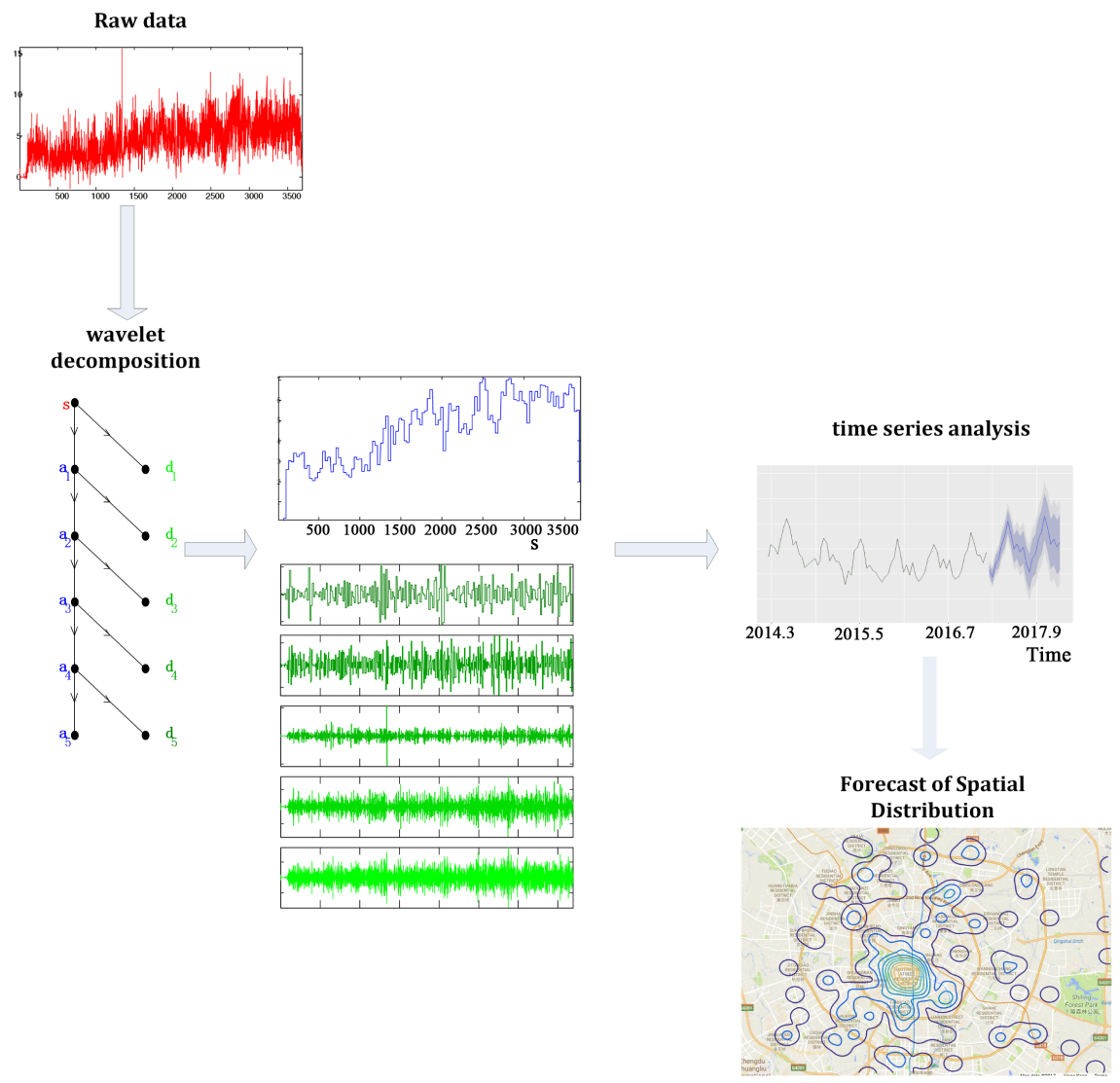

Figure 1. Wavelet analysis was performed on raw data representing the fluctuation of hospitalized patients. To identify the most representative of the fluctuations in the number of patients, ARIMA and seasonal ARIMA analysis was performed, and finally selected peak and trough time point for spatial distribution analysis and forecast. 


\section{Experimental (Mathematical Formulas, Tables and Equations)}

\subsection{Data Acquisition}

Data from Sichuan University West China Second Hospital from 2010-2015 were collected that contained patient's admissions time, address, attending physician name, reason for admission, diagnosis, and outpatient service check list. Patient names were de-identified with md5 encrypted desensitization. Patient communication address was anonymized using a key figure encrypted method.

\subsection{Seasonal Distribution Analysis Based on Wavelet Transform}

Count the segmented numbers of hospitalized children every 8 hours, and record the other information about admissions time, address, reason for admission, diagnosis into the comma-separated values (CSV) files. Using the Matlab wavelet toolbox to import the data, select Haar wavelet to decomposition, Select the best wavelet basis for compression at decomposition level 5 , and chose a proper threshold for de-noising. Exporting one main wave $\left(a_{1}\right)$, and 5 levels of wavelet $\left(d_{1}-d_{5}\right)$. Use the histogram for distribution observation. And the frequent pattern mining also is approached to find the law of the wavelet.

\subsection{Spatial Distribution}

Using the map of China's largest service providers, a map (NASDAQ: a map), the longitude and latitude of the patient can be obtained and the density can be calculated to estimate data distribution using Botev's method [1]. Specifically, using the front Haar wavelet decomposition results, we found selections deviating from Gaussian function biggest Haar decomposition level, and the level of the number of hospitalized patients as across-sectional observation unit. Using kernel density, the distribution function of the scales of hospitalized patients with spatial distribution were analyzed, and we gained influence on the fluctuation of the number of hospitalized patients with geographic location information. The basic principle of kernel density estimation algorithm for spatial data is as follows:

Kernel density estimation is a fundamental data smoothing problem where inferences about the population are made based on a finite data sample. In some fields such as signal processing and econometrics it is also termed the Parzen-Rosenbl at t-window method. This algorithm is often expressed as [1] [2],

$$
f_{h}(x)=\frac{1}{n} \sum_{i=1}^{n} K_{n}\left(x-x_{i}\right)=\frac{1}{n h} \sum_{i=1}^{n} K\left(\frac{x-x_{i}}{h}\right)
$$

where $\mathrm{K}(\bullet)$ is the kernel-a non-negative function that integrates to one and has mean zero-and $h>0$ is a smoothing parameter called the band width.

\subsection{Prediction and Machine Learning}

According to the analysis of wavelet transform in time domain and the nuclear 
density estimation on the space analysis, 5 years of pediatric admission data were extracted for different scales of fluctuation. Through the study of the spatial analysis of the data, we choose level 3 and level 2 of wavelet to fit the data distribution, which represent 8 and 15 day intervals, which respectively represent the weeks ( 7 days) and half month (15 days) of the time periods. We used ARIMA and linear regression fitting to make predictions about the future.

\section{Results and Discussion}

\subsection{Wavelet Transform}

On the time scale, patients admitted to hospital after the waveform can be divided into one mother wave and five wavelets (Figure 2 ) and the signal may be represented on every frequency band of the form $[f, 2 f]$ for all positive frequencies $f>0$. Then, the original signal can be reconstructed by a suitable integration over all the resulting frequency components. The frequency bands or subspaces (sub-bands) are scaled versions of a subspace at scale. Therefore, the following conclusions can be drawn from the above wavelet transform:

1) The whole surge of patients admitted was decomposed into one original (or mother) wavelet and 5 small wavelets.

2) Because 5 years is 1826 days, the Haar wavelet time period is derived from $2^{\wedge} \mathrm{n}(\mathrm{n}=1,2,3,4,5)$ days. The largest wavelet is about 32 days of the time period, which is almost one month.

3) The corresponding Mother wavelet should be the overall trend of hospital admissions, so that as time goes on, admissions increase.

4) The $5^{\text {th }}$ wavelet corresponds to admissions in days, which is almost a normal distribution (Figure 2).

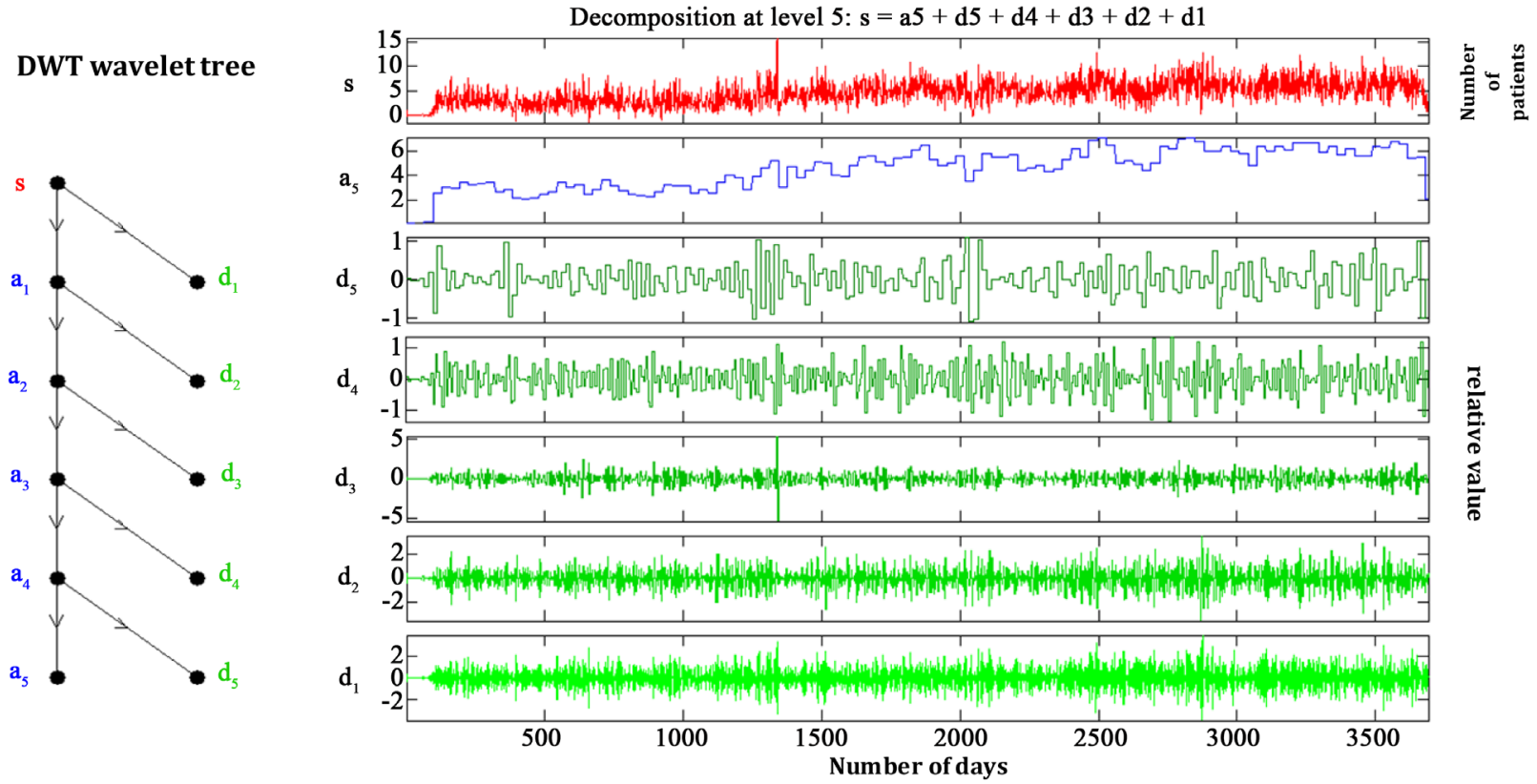

Figure 2. Wavelet transformation of hospitalized patient number. Wavelet analysis was performed on raw data representing the fluctuation. 


\subsection{Time and Space Distribution and Its Influencing Factors}

Using kernel density estimation, As the distance from the patient to the hospital gradually increased, the number of patients decreased exponentially, Using the Matlab $^{\oplus}$, CF tool toolbox, fit distance of patient from the hospital with the number of patients within these distance, we get the typical fitting formula as follows:

$$
f(x)=0.595 * x^{-0.0026}
$$

where $f(x)$ is the patients number, and $\mathrm{x}$ is the distance to West China Hospital, the correlation coefficient $\left(\mathrm{R}^{\wedge} 2\right)$ is 0.875 (Figure 3$)$. Thus, hospitalized patients will gradually decrease with distance. Using wavelet transform, we obtained the wavelet scale for patients with the distribution fitting, from Haar wavelet from 1 - 5. The smallest scale wavelet (wavelet 5) can be considered as a random white noise, and there is no clear trend for the distribution of patient space in this scale. And with the scale, the third wavelets, second wavelets, it can be viewed as the monthly and seasonal distribution. Therefore, the month and season can be used as a larger distribution cycle to test the source of the patient's space distribution in Chengdu, in Sichuan, or in China (Figure 2).

\subsection{Forecast for Patient Number}

The ARIMA the forecast model suggests 3500 - 4700 patients per month, it will be described as follows (Figure 4 and Table 1 ). And under the short-term modulation of patient's number, wave 3 represents seasonal patterns of patient surges, so we used seasonal ARIMA models (SAM) to represent patients trend changes [7]. These data appear in Table 2.

\subsection{Effect of Seasonal Rotation to Diseases and the Prediction Model}

Classical ARIMA models are typically well-suited for short-term forecasts, but not for longer term forecasts due to the convergence of the autoregressive com-

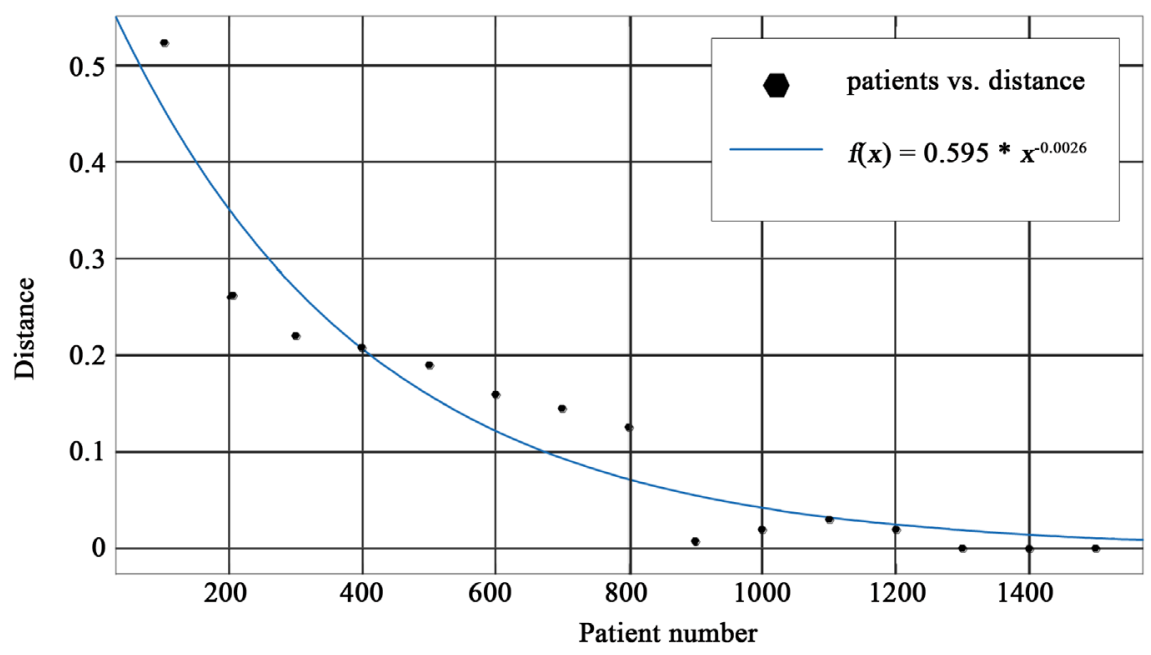

Figure 3. With the increase in the distance of the patient's residence, the number of patients gradually reduced. 


\section{Forecasts from BAT model}

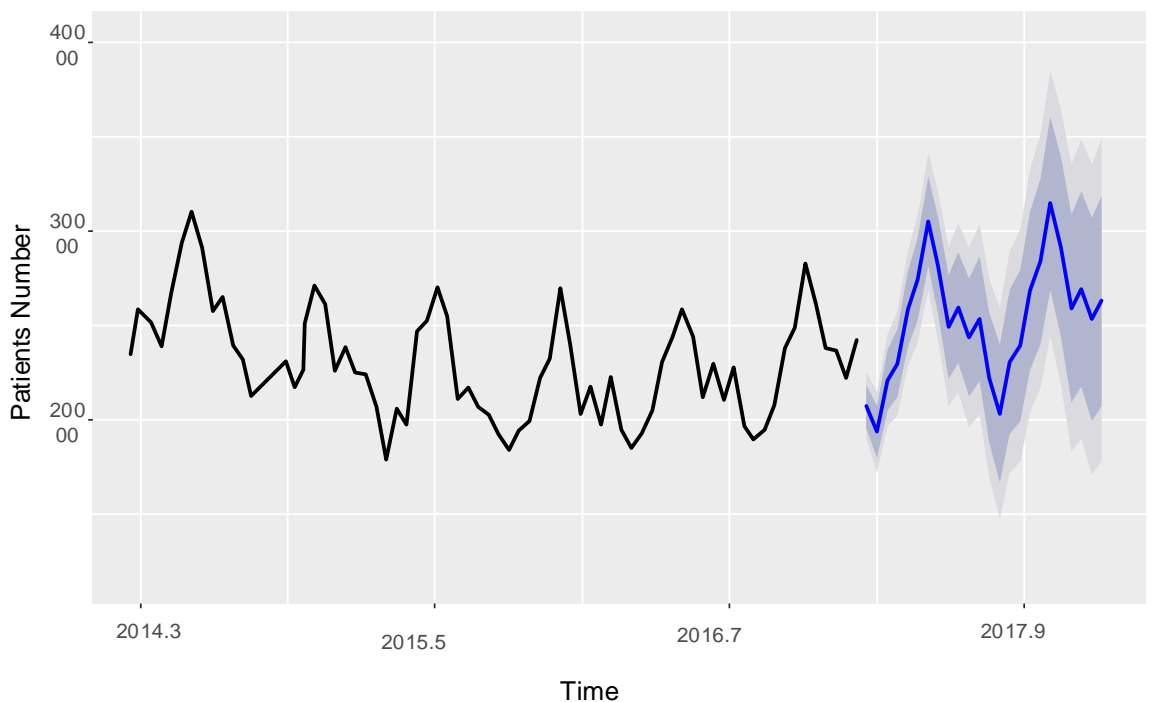

Figure 4. The fluctuation of patient number from Mar. 2014 to Dec. 2016 and the curve with shadow is on behalf of the forecast of volatility of patient number in future fitted by ARIMA and seasonal ARIMA.

Table 1. The results of the fitting.

\begin{tabular}{ccc}
\hline & \multicolumn{2}{c}{ Value } \\
\hline Coefficients & $\mathrm{a}=$ & $0.5954(0.4597,0.7311)$ \\
(with 95\% confidence bounds) & $\mathrm{b}=$ & $0.002643(0.003389,0.001896)$ \\
SSE: 0.02698 & SSE: & 0.02698 \\
R-square: 0.9078 & R-square: & 0.9078 \\
Adjusted: 0.9007 & Adjusted: & 0.9007 \\
& RMSE: & 0.04556
\end{tabular}

Table 2. ARIMA forecast result.

\begin{tabular}{ccc}
\hline & Ma1 & Sma1 \\
\hline & -0.4303 & -0.5528 \\
s.e. & 0.1228 & 0.1784 \\
\hline
\end{tabular}

ponent of the model to the mean of the time series. However, as mentioned above, the use of too short a time cycle causes random fluctuations. Therefore, the selection of a reasonable statistical cycle for correct statistical conclusions is essential.

However, according to the multi-scale theory, one random process under the one view can change the organized characteristic in other scales and show corresponding laws. So, tools are needed to observe the time series in different scales. Wavelet analysis is suitable for efficiency in time [7] [8], or frequency (as harmonic, Shannon, Meyer) [9] [10] domain. In the time distribution, and the present random fluctuations inpatient admissions, using wavelet decomposition, 
patients with fluctuations in different scales have different rules. We calculate the correlation between GDP and the number of patients by at a specific point in time, the formula is:

$$
R=\frac{\sum\left(X_{i}-\bar{X}\right)\left(Y_{i}-\bar{Y}\right)}{\sqrt{\sum\left(X_{i}-\bar{X}\right)^{2}\left(Y_{i}-\bar{Y}\right)^{2}}}
$$

where $X_{i}$ represents the number of patients in the $i$-th period, $Y_{i}$ represents the GDP announced by the Chinese government at the end of the $i$-th period

From the scale of the mother wave, growth in admissions increased over time in the manner of an accelerating index increase. This increase was closely related to China's GDP growth (Figure 5$).(R=0.8826)$. ARIMA model can be used to predict future admissions. From $2^{\text {nd }}$ and $3^{\text {rd }}$ scale, the two scale can reflect the number of patients on the monthly and quarterly changes, which is true for pediatrics. In autumn, patient admissions will decrease because of the school season. Seasonal ARIMA models suggest that outpatients since the end of 2014 declined (Figure 4), suggesting that the classification system of the hospital may contribute to the patient count. However, this cannot be modeled with a classical linear ARIMA fitting. With the ARIMA model the existing classification system of hospital patient decline due to seasonal and monthly differences did not have big fluctuations, and in 2017 the rebound was greater after October. This rebound needs attention (Figure 4).

\subsection{The Spatial Distribution of Patients and Its Influencing Factors}

Segmentation fitting shows that the number of hospitalized patients will decrease exponentially with distance from the hospital, and these data agree with Wang's group [11]. Specific to the urban area of Chengdu, after the implementation of hierarchical treatment in 2015, patients in the urban distribution changed significantly. Patients decreased with increasing distance from the hos-

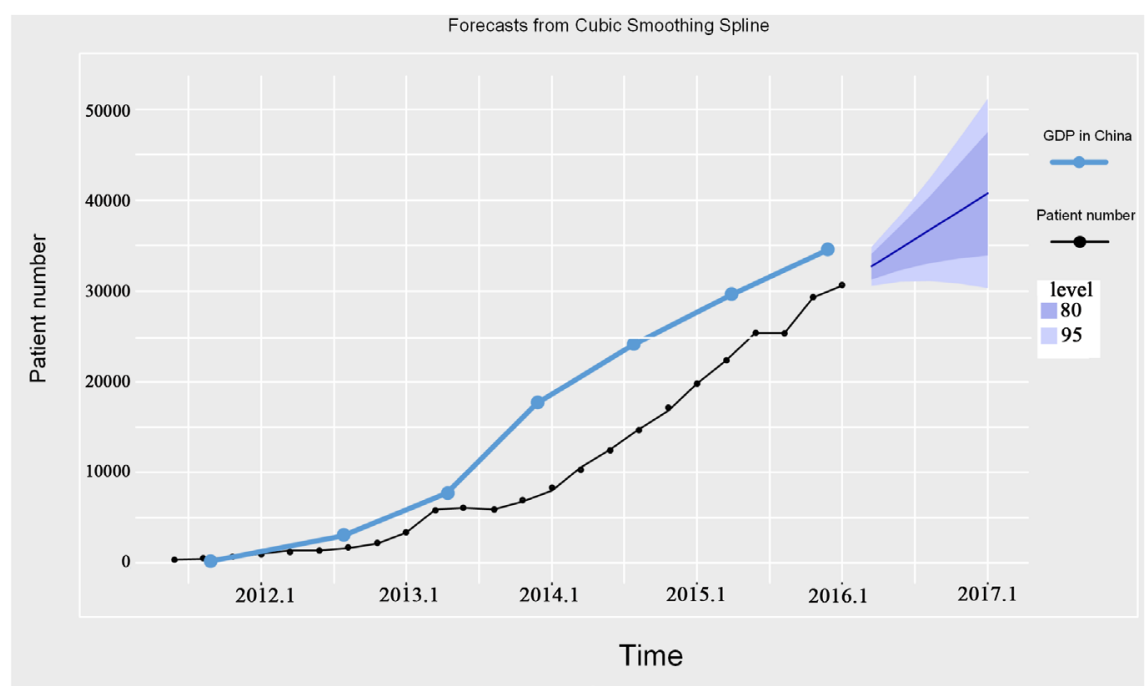

Figure 5. The patient number will increase with the GDP growth. 
pital, and this decrease was exponentially distributed. The relationship with time is clear, too, and it indicates that patients from outer suburbs to the hospital primarily by time constraints. Elective patients will generally avoid peak enrollment in September. Spatial density analysis showed that after the implementation of hierarchical diagnosis and treatment, distribution patient patterns change. In September 2015, hospitalized patient distribution is more concentrated than that from September 2014, indicating that the change in spatial distribution of the diagnosis and treatment system has been identified by the KDE (kernel density estimation) algorithm (Figure 6).

\section{Conclusions}

Multi-scale phenomena can be found in numerous natural events that occur over long time periods and within wide spaces. Multi-scale effects can be analysed using multi-scale effects for complex fluctuations caused by complex factors. As can be seen from our study, the number of growing patients presents some seasonal cyclical fluctuations and shows a trend of gradually increase. This spatial and temporal fluctuation poses a double challenge to the allocation of medical resources. In order to cope with this dual challenge, it is advisable to increase the supply of medical care in the surrounding area during the busy season, where most patients with mild symptoms are able to seek medical attention nearby. In the winter and summer school vacation, hospital should increase the supply of elective outpatient clinics.

Multi-scale phenomena can be realized with mathematical microscope-wavelet analysis. Through wavelet analysis and other predictive methods such as ARIMA, we analyse the time and spatial distribution of patients in a Chinese hospital over a five-year period. Data show that even a patient address can be useful information. The idea of data mining based on spectral analysis and spatial distribution can be extended to many aspects of social management such as education resource assessment, medical resources distribution assessment and future social resources planning.

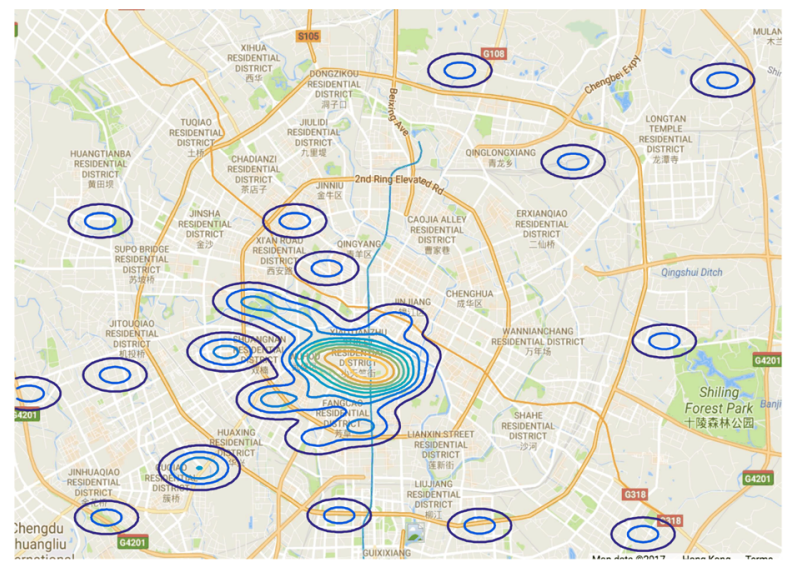

2014 Sep

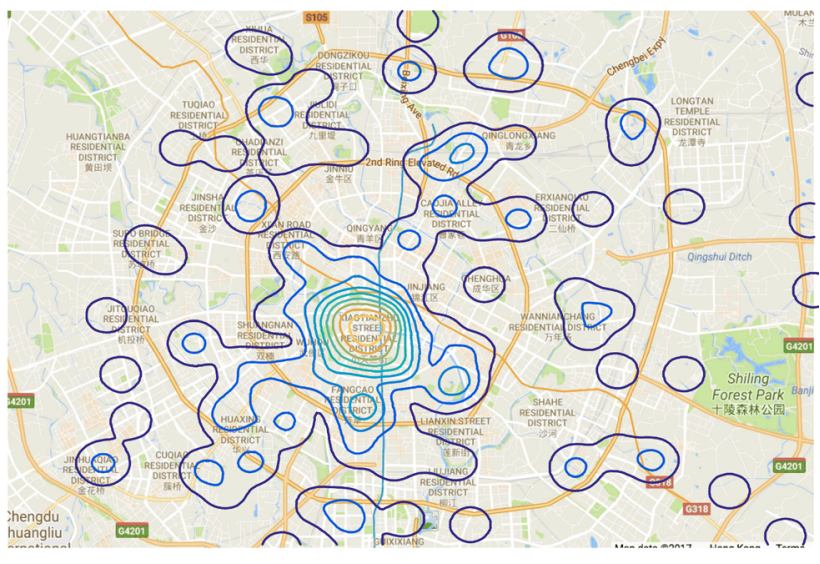

2015 Sep

Figure 6. The spatial distribution of patient in downtown of Chengdu in 2014 and 2015. 


\section{Acknowledgements}

This project was supported by the funds of Strategic Innovation Funding of Science and Technology of SiChuan University. (No. 20160040321).

\section{References}

[1] Bayram, J.D., Sauer, L., Catlett, C.L., Levin, S., Cole, G., Kirsch, T.D., et al. (2013) Critical Resources for Hospital Surge Capacity: An Expert Consensus Panel. PLoS Currents, 5. https://doi.org/10.1371/currents.dis.67c1afe8d78ac2ab0ea52319eb119688

[2] Hung, K.V. and Huyen, L.T. (2011) Education Influence in Traffic Safety: A Case Study in Vietnam. IATSS Research, 34, 87-93. https://doi.org/10.1016/j.iatssr.2011.01.004

[3] La, Q.N., Lee, A.H., Meuleners, L.B. and van Duong, D. (2013) Prevalence and Factors Associated with Road Traffic Crash among Taxi Drivers in Hanoi, Vietnam. Accident Analysis \& Prevention, 50, 451-455. https://doi.org/10.1016/j.aap.2012.05.022

[4] Toor, M.R., Singla, A., Devita, M.V. and Michelis, M.F. (2014) Characteristics, Therapies, and Factors Influencing Outcomes of Hospitalized Hypernatremic Geriatric Patients. International Urology and Nephrology, 46, 1589-1594. https://doi.org/10.1007/s11255-014-0721-2

[5] Koberich, S. and Farin, E. (2016) Factors Influencing Hospitalized Patients' Perception of Individualized Nursing Care: A Cross-Sectional Study. BMC Nursing, 15, 1 11. https://doi.org/10.1186/s12912-016-0137-7

[6] Livera, A.M.D., Hyndman, R.J. and Snyder, R.D. (2009) Forecasting Time Series with Complex Seasonal Patterns Using Exponential Smoothing. Monash Econometrics \& Business Statistics Working Papers, 106, 1513-1527.

[7] Chen, C.F. and Hsiao, C.H. (1997) Haar Wavelet Method for Solving Lumped and Distributed-Parameter Systems. IEE Proceedings-Control Theory and Applications, 144, 87-94. https://doi.org/10.1049/ip-cta:19970702

[8] Lewis, A.S. and Knowles, G. (1991) VLSI Architecture for 2D Daubechies Wavelet Transform without Multipliers. Electronics Letters, 27, 171-173. https://doi.org/10.1049/el:19910110

[9] Meyer, Y. (1991) Wavelets: Algorithms and Applications. Society for Industrial \& Applied Mathematics, Philadelphia.

[10] Shannon, C.E. (1974) A Mathematical Theory of Communication. McGraw-Hill, New York.

[11] Wang, F. and Wei, L. (2005) Assessing Spatial and Nonspatial Factors for Healthcare Access: Towards an Integrated Approach to Defining Health Professional Shortage Areas. Health \& Place, 11, 131-146. https://doi.org/10.1016/j.healthplace.2004.02.003 
Submit or recommend next manuscript to SCIRP and we will provide best service for you:

Accepting pre-submission inquiries through Email, Facebook, LinkedIn, Twitter, etc. A wide selection of journals (inclusive of 9 subjects, more than 200 journals)

Providing 24-hour high-quality service

User-friendly online submission system

Fair and swift peer-review system

Efficient typesetting and proofreading procedure

Display of the result of downloads and visits, as well as the number of cited articles Maximum dissemination of your research work

Submit your manuscript at: http://papersubmission.scirp.org/

Or contact jgis@scirp.org 